\title{
An Analysis of the Development and Present Situation of Children's Psychological Counseling and Treatment
}

\author{
Xiaozhen Liu ${ }^{1, a}$, Yanchun $\mathrm{Qi}^{2, \mathrm{~b}}$ \\ ${ }^{1}$ Gannan Medical University, Ganzhou, Jiangxi, 341000 \\ ${ }^{a}$ email, ${ }^{b}$ email
}

Keywords: Children Psychological Counseling and Treatment, Development Status

\begin{abstract}
Compared with the development of psychological counseling and treatment in adults, children's psychological counseling and treatment history is relatively short, in addition to the diagnosis of children with mental retardation, other children's psychological counseling and treatment literature can only be traced back to the beginning of this century. Children's psychological counseling and treatment development and the following events are closely related.
\end{abstract}

\section{Introduction}

With the development of Chinese society, the improvement of people's living standards, people have made more and more intense demand for mental health. People are increasingly aware that improving the psychological quality of the whole nation is an important part of the construction of spiritual civilization. Psychological counseling is the beginning of universal attention to the product of spiritual life. As a background, in recent years, psychological counseling and treatment in China has a considerable degree of development, and show some prominent development characteristics.

Psychological counseling and psychological treatment in the theoretical basis, work forms and so there are many overlapping, so sometimes not distinguish. The distinction between the two experts generally believe that psychological counseling mainly to solve the health and sub-health groups of psychological problems; and psychological treatment mainly in the presence of mental illness in the crowd. However, in the actual operation, because the seekers often can not distinguish between whether they are sick, so the two often overlap.

\section{Children's Psychological Counseling and Treatment Characteristics}

Children is in the rapid development and change of the state, has not yet formed a complete, mature self-awareness, cognitive ability is limited, so children's psychological counseling and treatment have the following aspects of the characteristics.

First, from the development point of view, the children for consultation and treatment. Children are experiencing rapid changes in physiological, cognitive, social and emotional, and the diagnosis of child disorders is more dependent on age and developmental factors. For example, enuresis is completely different from the meaning of a 2-year-old child and a 10-year-old child, and there is a need for a clear understanding of the course of change or the basic level of a particular behavior or symptom at different ages and levels of development, so as to decide whether to give treatment. Children in the process of development, behavior has undergone great changes, the behavior of different age problems sometimes obvious, sometimes disappear, usually in the development process, the problem behavior is gradually reduced, but there may be some clinical problems, at different ages Replaced by another act. Such as children are used to threaten, scare other children, to adolescence will continue this aggressive behavior, but may be expressed in different ways, such as fighting, fighting and so on. In addition, childhood children, who have not yet formed autonomous self-concept, lack a reference system for treating reality, possibilities and values, and thus they are more difficult to cope with stress events than adolescents and adults, and because of their dependence on adults So that they are in the face of rejection, failure vulnerable to injury.

Second, the vast majority of children do not require their own treatment, usually by adults to counseling, treatment clinics, therefore, adults in the treatment arrangements, the assessment of 
children's problems and the entire treatment process plays an important role. Children usually do not think they are "patients" with emotional or behavioral disorders, and to adolescence, they may think that the problem is caused by environmental, social, family and parental problems, so that the application of various intervention methods can be difficult, And children can adhere to treatment is also in doubt.

Thirdly, the problem of children is very extensive, and sometimes children are the focus of treatment, and sometimes there are other problems. Such as children are victims of physical or sexual abuse, or children's problems are family events (such as parental divorce). Therefore, it is difficult to find a child problem, because the child is not good at or unable to seek external help, parents may deliberately conceal some facts. Sometimes, the problem of children is due to parents or teachers inadvertently high expectations or parents of their own problems such as depression, anxiety caused, therefore, as needed, please parents, teachers, co-participation in treatment.

Fourth, due to the different age and different levels of development of children, the treatment of children usually includes conversation, games, reward new behavior, practice activities, etc., for children who have not yet fully grasp the language, you can also use a variety of adjuvant therapy tools, such as Dolls, stories, pictures and so on. It is unlikely that they will only be treated for verbal communication.

\section{Children's Psychological Counseling and Treatment Methods}

A salient feature of game therapy is that the therapist has little interest in the outcome of the game (such as the game that promotes cognitive development). They attach importance to the meaning of game behavior, attention to the game in the process of children expressed by their emotional, psychological-related language significance. As game therapy is suitable for children's development, it is particularly suitable for younger children. A variety of models for the treatment of children can also use the game to achieve its therapeutic goals. Such as storytelling techniques, so that children tell stories, therapists through the repetition of children's stories, adding more healthy adaptation of the content to influence children's awareness, so that children in real life have a better choice. Another example is the fair game therapy, through the treatment of children in the performance of the game to make a fair and honest response, rather than blindly accept to help children understand their own interpersonal responsibilities. Game therapy combined with other methods, but also produced a group game therapy, family game therapy and other methods. Allen believes that game therapy can be divided into two categories, one emphasizes the content of the game, a class emphasizes the treatment relationship. The former used the game as a tool for digging children's unconscious desire or fantasy, so let the children express and release feelings through the game. The latter uses the game as a tool for the caregiver to establish a good relationship with the child, and it is important to use the game to establish the relationship than the actual content of the game. Although the different methods of emphasis on different methods, but the game treatment goals summarized mainly in the following three aspects: the first is to understand the symptoms of children; the second is to reduce the symptoms; the third is to promote children to obtain a positive self-concept and the people, Positive knowledge of the environment.

Behavioral maladjustment mainly refers to attack behavior, lying, antisocial behavior and so on. The specific techniques used to correct the behavioral disorders are: "Operational conditional techniques". First select the target behavior, which includes the behavior to be reduced and to encourage increased behavior. Such as reward good behavior, mainly pro-social behavior. Those who do not expect the behavior did not occur, but also to strengthen. Before treatment, the first assessment of the incidence of adverse behavior, and then the implementation of positive, is to strengthen that when the desired behavior appears or increase, do not expect the behavior did not occur when the praise, reward. If you are using positive reinforcement, but children have not observed changes in behavior within days, you may consider replacing the reinforcement. Studies have shown that the use of social reinforcement alone, the role of aggressive sexual behavior of children is not the ideal way is to praise and material incentives combined. "Coin" is also a way to correct aggressive behavior. Before the implementation of the tokens, the first choice of target 
behavior, reverse reinforcement (with token can be exchanged for the reinforcement) and tokens; and then in consultation with children to develop tokens exchange system; tell children what kind of behavior can be obtained one or several The tokens are given immediately after the appearance of the desired behavior; tell the child how many tokens can be exchanged for the corresponding reverse reinforcement; the time and place of the agreed exchange.

Family therapy began in the 1950s. Family therapy has two basic points of view. One is the theory of systematic family therapy. The theory that any child's symptoms are not only children's own problems, children are a member of the family, is the whole "system" part, in a "system", the children's words and deeds continue to affect the people around, But also by the other members of the family, therefore, in the psychological treatment of children, in addition to understand the symptoms of children themselves, but also to understand the behavior of children, emotional problems occur throughout the background environment, and these environments and children Of the interaction, the treatment should be carried out for the whole family, that is, in the correction of children at the same time, we must change the whole system. Since children are completely dependent on their families to meet their basic needs, they can use games and other child-oriented technologies to improve intra-family system levels of communication. Another point of view of family therapy is behavioral theory. The theory suggests that changing parental responses to children by changing the behavior of children, such as parental management training, is a technique of family behavioral therapy. In recent years, family therapy has joined the point of view, emphasizing the family's attribution, attitude, expectations and emotions.

\section{Development Direction and Enlightenment of Children's Psychological Counseling and Its Treatment}

Traditional pediatric psychological therapy is divided into two parts: affective disorder and behavioral disorder. In fact, it is easy for us to question this sub-law: the degree of aggregation of children, whether in anxiety or in the measure of depression, is not enough; the covariance between behavioral and affective disorders is high. If you want to study a pure depressed child's case, the researchers screen out those who have depressed characteristics of children. However, in clinical samples, many of the therapists found depressed atypical children. So, this type of division is not appropriate. The intervention of the ecological model does not classify the treatment target. It is not only widely applicable to the psychological behavior of children, but also applies to children's anxiety and depression and other emotional disorders. And children's psychological counseling and treatment practice in the use of emotional disorders is relatively small, it will be an important future development direction.

Ecological model of child psychotherapists need to master a number of areas of knowledge and skills, including adult mental health "social work" learning psychology "career counseling" psychiatry. Therapists should also distinguish between the following areas and the difference between therapy: children and family therapy "school counseling" marriage therapy "psychiatric treatment" adult therapy "career counseling.

After World War II, many clinical research and psychiatric experts on children's mental health survey showed that children's mental health problems and the rapid increase in the use of psychotropic drugs, the community needs a variety of mental health services. At present, the public investment in mental health services is mainly in the diagnosis of children, in the treatment of inadequate investment. The establishment of ecological models, for the family will be more expensive. However, this service model is more cost-effective because its effect is significant. Therefore, in the application areas must strengthen the children's mental health ecological model of public service.

\section{Conclusion}

Children's psychotherapy is very different from adults, many of the problems of children are development problems, and some problems are reflected in the family problem, in the consultation 
and treatment of children, the diagnosis is very important. Currently used for children's counseling and treatment methods are mostly derived from the treatment of adults, and thus have some limitations. In the future, child psychologists and clinical psychologists will be asked to study and create a more appropriate treatment for children. At present, a new discipline - the development of psychopathology has embodied this combination. Child psychologist brownfin Brenner and other scholars suggest that the social ecology system for children's development should include the family system, the peer relationship system, the community and the school system, which have a direct impact on the development of children, children's clinical psychology The family should draw on this view, from the perspective of the ecosystem, to develop prevention and intervention programs and measures.

\section{References}

[1] Tao Jinhua, Yao Benxian. Study on the Present Situation of Individual Psychological Counseling in Colleges and Universities [J]. Journal of Hygiene Research in China, 2015 (10)

[2] Li Guojun. Talking about the development of psychological counselors and countermeasures [J]. Human Resources Development, 2014 (17)

[3] Liu Zhengkui, Wu Kankan, Zhang Kan. China's major natural disasters after the psychological assistance to explore and challenge [J]. China Soft Science, 2011 (05)

[4] Qian Mingyi, Chen Ruiyun, Zhang Lili, Zhang Zhi-Feng. Study on the future demand of psychological counseling therapists in China [J]. Chinese Journal of Mental Health, 2010 (12)

[5] Zhang Zhifeng, Yi Chunli, Qian Mingyi, Zhong Jie. Comparison of management of mental health service institutions in medical and educational fields [J]. Chinese Journal of Clinical Psychology, 2009 (06) 